\section{TATRA \\ MOUNTaiNS \\ Mathematical Publications}

DOI: $10.2478 / \mathrm{tmmp}-2021-0030$

Tatra Mt. Math. Publ. 80 (2021), 35-52

\title{
SOLVING NONLINEAR VOLTERRA-FREDHOLM INTEGRAL EQUATIONS USING AN ACCURATE SPECTRAL COLLOCATION METHOD
}

\author{
Fatima Hamani ${ }^{1}$ - Azedine Rahmoune ${ }^{2}$ \\ ${ }^{1}$ Department of mathematics, University of M'sila, 28000, ALGERIA \\ ${ }^{2}$ Department of mathematics, University of Bordj Bou Arreridj, El Anasser, 34030, ALGERIA
}

\begin{abstract}
In this paper, we present a Jacobi spectral collocation method to solve nonlinear Volterra-Fredholm integral equations with smooth kernels. The main idea in this approach is to convert the original problem into an equivalent one through appropriate variable transformations so that the resulting equation can be accurately solved using spectral collocation at the Jacobi-Gauss points. The convergence and error analysis are discussed for both $L^{\infty}$ and weighted $L^{2}$ norms. We confirm the theoretical prediction of the exponential rate of convergence by the numerical results which are compared with well-known methods.
\end{abstract}

\section{Introduction}

We consider the nonlinear Volterra-Fredholm integral equations (VFIEs) given by the general form:

$$
u(s)=g(s)+\int_{0}^{s} V(s, t) \psi_{1}(u(t)) d t+\int_{0}^{1} F(s, t) \psi_{2}(u(t)) d t, \quad s \in[0,1],
$$

where the kernels $V, F$, and $g, \psi_{1}, \psi_{2}$, are given smooth functions about their variables, and $u(s)$ is the unknown function to be determined. Volterra-Fredholm integral equations arise in a variety of applications in many fields, including potential theory, electricity and magnetism, antenna synthesis problem, communication theory, mathematical economics, etc. However, in practice, the VFIEs

(C) 2021 Mathematical Institute, Slovak Academy of Sciences.

2010 Mathematics Subject Classification: 45G10, 33C45, 41A55.

Keywords: Jacobi collocation methods, convergence analysis, spectral methods, nonlinear Volterra-Fredholm integral equations.

(c) (1) () $९$ Licensed under the Creative Commons BY-NC-ND 4.0 International Public License. 
are too difficult to solve analytically. For this reason it is interesting to develop a new accurate approximation technique to solve them numerically. Many authors focussed on the development of numerical methods for the general mixed integral equations. For instance, among those interested in Equation (11), Yousefi and Razzaghi 21] presented a Legendre wavelet approximation method, Babolian et al. 3] presented a computational method based on replacement of the unknown function by truncated series of well-known Chebyshev expansion of functions. In paper [16] authors developed a collocation method using rationalized Haar functions to solve the special form of Equation (1). Marzban et al. [13] presented a composite collocation method based on Hybrid of Block-Pulse functions and Lagrange polynomials. Thereafter, a computational method based on Bernstein operational matrices to the same equation was presented in [1]. Very recently, Amiri et al. 1] developed a spectral collocation method with piecewise trigonometric basis functions.

It is well-known that the classical Jacobi polynomials and their special cases have been used extensively in mathematical analysis and applications (see, e.g., [2, 5, 8, 9, 19, 20] and reference therein). Thus, the main aim of the present work is to develop an accurate spectral method based on these useful generating sets of orthogonal polynomials to solve Eq. (10). First, we must transform the problem set in the given interval to $[-1,1]$ by means of appropriate variable transformations. Then, we apply the standard spectral Jacobi-collocation method to the resulting equation in which Jacobi-Gauss points are utilized together with Legendre-Gauss quadrature to reduce it to the solution of nonlinear equations.

The remainder of this paper is organized as follows. In Section 2 , we present the Jacobi spectral collocation method for nonlinear Volterra-Fredholm integral equations (11). In Section 3. we get the error estimates in both $L^{\infty}$ and $L_{w}^{2}$ norms. In Section 4. we present some numerical examples and we compare our results with well-known methods to appear the superiority of the present approach.

\section{Spectral Jacobi-collocation method}

In this section, we will discuss the Jacobi spectral collocation method to solve Eq. (11). Before proceeding further, we need to introduce the following notations, let $I=[-1,1]$, and $w^{\alpha, \beta}(x)=(1-x)^{\alpha}(1+x)^{\beta}$ be a weight function, for $\alpha, \beta>-1$. Then we use the notation $L_{w^{\alpha, \beta}}^{2}(I)$ to denote the set of all square integrable 
functions associated with the weight function $w^{\alpha, \beta}$ equipped with the norm

$$
\|v\|_{w^{\alpha, \beta}}=\left(\int_{-1}^{1}|v(x)|^{2} w^{\alpha, \beta}(x) d x\right)^{1 / 2} .
$$

For a given positive integer $N$, we denote by $\left\{\sigma_{N, j}^{\alpha, \beta}\right\}_{j=0}^{N}$ the points of the Gauss-Jacobi quadrature formula, which are the roots of the Jacobi polynomials $J_{N+1}^{\alpha, \beta}$.

Let $\mathcal{C}(I)$ denotes the set of all continuous functions on $I$, equipped with the norm

$$
\|v\|_{\infty}=\max _{x \in I}|v(x)|
$$

and $\mathcal{P}_{N}$ the space of all polynomials of degree not more than $N$. For any $v \in \mathcal{C}(I)$ we can define the Lagrange interpolating polynomial $I_{N}^{\alpha, \beta} v \in \mathcal{P}_{N}$ as

$$
I_{N}^{\alpha, \beta} v(x)=\sum_{j=0}^{N} v\left(\sigma_{N, j}^{\alpha, \beta}\right) L_{j}(x),
$$

where $\left\{L_{j}\right\}_{j=0}^{N}$ is the Lagrange interpolation basis function associated with the Jacobi collocation points $\left\{\sigma_{N, j}^{\alpha, \beta}\right\}_{j=0}^{N}$.

For convenience, we consider the following variable transformations

$$
s=\frac{x+1}{2}, \quad t=\frac{y+1}{2}, \quad x, y \in I,
$$

so that Eq. (11) becomes

$$
U(x)=G(x)+\int_{-1}^{x} \bar{V}(x, y) \psi_{1}(U(y)) d y+\int_{-1}^{1} \bar{F}(x, y) \psi_{2}(U(y)) d y,
$$

where

$$
\begin{gathered}
U(x)=u\left(\frac{x+1}{2}\right), \\
G(x)=g\left(\frac{x+1}{2}\right), \\
\bar{V}(x, y)=\frac{1}{2} V\left(\frac{x+1}{2}, \frac{y+1}{2}\right), \\
\bar{F}(x, y)=\frac{1}{2} F\left(\frac{x+1}{2}, \frac{y+1}{2}\right) .
\end{gathered}
$$

Then we introduce the linear transformation

$$
y=y(x, \theta)=\frac{x+1}{2} \theta+\frac{x-1}{2}, \quad x, \theta \in I,
$$




\section{F. HAMANI - A. RAHMOUNE}

which transfers the first integral term in (2) to the form

$$
\int_{-1}^{x} \bar{V}(x, y) \psi_{1}(U(y)) d y=\frac{x+1}{2} \int_{-1}^{1} \bar{V}(x, y(x, \theta)) \psi_{1}(U(y(x, \theta))) d \theta .
$$

Hence, Eq. (2) becomes

where

$$
U(x)=G(x)+\int_{-1}^{1} \widetilde{V}(x, \theta) \psi_{1}(U(y(x, \theta))) d \theta+\int_{-1}^{1} \widetilde{F}(x, \theta) \psi_{2}(U(\theta)) d \theta
$$

$$
\widetilde{V}(x, \theta)=\frac{x+1}{2} \bar{V}(x, y(x, \theta)), \quad \widetilde{F}(x, \theta)=\bar{F}(x, \theta) .
$$

The spectral Jacobi-collocation method for solving Eq. (11) is to seek an approximate solution $U_{N}(x) \in \mathcal{P}_{N}$, such that $U_{N}(x)$ satisfies Eq. (3) at the collocation points $\sigma_{N, j}^{\alpha, \beta}$, i.e.,

$$
\begin{array}{r}
U_{N}\left(\sigma_{N, j}^{\alpha, \beta}\right)=G\left(\sigma_{N, j}^{\alpha, \beta}\right)+\int_{-1}^{1} \widetilde{V}\left(\sigma_{N, j}^{\alpha, \beta}, \theta\right) \psi_{1}\left(U_{N}\left(y_{N, j}^{\alpha, \beta}(\theta)\right)\right) d \theta+ \\
\int_{-1}^{1} \widetilde{F}\left(\sigma_{N, j}^{\alpha, \beta}, \theta\right) \psi_{2}\left(U_{N}(\theta)\right) d \theta
\end{array}
$$

where $y_{N, j}^{\alpha, \beta}(\theta):=y\left(\sigma_{N, j}^{\alpha, \beta}, \theta\right)$. The integral terms in the above equation can be accurately approximated by using Legendre-Gauss quadrature formula. Let $\left\{\theta_{k}, \omega_{k}\right\}_{k=0}^{N}$ be the Legendre-Gauss nodes and weights, and there holds

$$
\begin{gathered}
\int_{-1}^{1} \widetilde{V}\left(\sigma_{N, j}^{\alpha, \beta}, \theta\right) \psi_{1}\left(U_{N}\left(y_{N, j}^{\alpha, \beta}(\theta)\right)\right) d \theta \\
\int_{-1}^{1} \widetilde{F}\left(\sigma_{N=0}^{\alpha, \beta}, \theta\right) \psi_{2}\left(U_{N}(\theta)\right) d \theta \\
\left.\sim \sum_{k=0}^{N} \omega_{k} \widetilde{F}\left(\sigma_{N, j}^{\alpha, \beta}, \theta_{k}\right) \psi_{1}\left(U_{N}\left(y_{N, j}^{\alpha, \beta}, \theta_{k}\right) \psi_{2}\left(\theta_{k}\right)\right)\right)
\end{gathered}
$$

Next, by using the Lagrange interpolation to approximate the nonlinear parts in (5]) and (6), namely,

$$
\begin{gathered}
\psi_{1}\left(U_{N}\left(y_{N, j}^{\alpha, \beta}(\theta)\right)\right) \sim I_{N}^{\alpha, \beta}\left(\psi_{1}\left(U_{N}\left(y_{N, j}^{\alpha, \beta}(\theta)\right)\right)\right)=\sum_{j=0}^{N} \psi_{1}\left(\hat{U}_{N, j}^{\alpha, \beta}\right) L_{j}\left(y_{N, j}^{\alpha, \beta}(\theta)\right), \\
\psi_{2}\left(U_{N}(\theta)\right) \sim I_{N}^{\alpha, \beta}\left(\psi_{2}\left(U_{N}(\theta)\right)\right)=\sum_{j=0}^{N} \psi_{2}\left(\hat{U}_{N, j}^{\alpha, \beta}\right) L_{j}(\theta)
\end{gathered}
$$

where

$$
\hat{U}_{N, j}^{\alpha, \beta}:=U_{N}\left(\sigma_{N, j}^{\alpha, \beta}\right)
$$


we get the full collocation scheme

$$
\begin{aligned}
\hat{U}_{N, j}^{\alpha, \beta}=G\left(\sigma_{N, j}^{\alpha, \beta}\right)+\sum_{k=0}^{N} \omega_{k} \widetilde{V}\left(\sigma_{N, j}^{\alpha, \beta}, \theta_{k}\right) I_{N}^{\alpha, \beta}\left(\psi_{1}\left(U_{N}\left(y_{N, j}^{\alpha, \beta}\left(\theta_{k}\right)\right)\right)\right)+ \\
\\
\sum_{k=0}^{N} \omega_{k} \widetilde{F}\left(\sigma_{N, j}^{\alpha, \beta}, \theta_{k}\right) I_{N}^{\alpha, \beta}\left(\psi_{2}\left(U_{N}\left(\theta_{k}\right)\right)\right) .
\end{aligned}
$$

We can get the values of $\hat{U}_{N, j}^{\alpha, \beta}, j=0,1, \ldots, N$, by solving (17). An approximate solution of Eq. (2) will be given by $U(x) \sim U_{N}(x)=\sum_{j=0}^{N} \hat{U}_{N, j}^{\alpha, \beta} L_{j}(x)$, since the exact solution of the original equation (11) can be written as

$$
u(s)=U(x), \quad \text { where } \quad x=2 s-1, \quad s, x \in I .
$$

\section{Error estimates}

Before presenting error estimates for the above scheme, we need to introduce the following weighted Hilbert space with some useful lemmas.

For a nonnegative integer $m$, we define

$$
H_{w}^{m}(I)=\left\{v: \partial_{x}^{k} v \in L_{w^{\alpha, \beta}}^{2}(I), 0 \leq k \leq m\right\},
$$

equipped with the following semi-norm and norm

$$
|v|_{k, w}=\left\|\partial_{x}^{k} v\right\|_{w^{\alpha, \beta}}, \quad\|v\|_{m, w}=\left(\sum_{k=0}^{m}|v|_{k, w}^{2}\right)^{1 / 2} .
$$

Also, it is convenient to introduce the semi-norms

$$
|v|_{H_{w}^{m}(I)}=\left(\sum_{k=\min (m, N+1)}^{m}|v|_{k, w}^{2}\right)^{1 / 2} .
$$

For $k \in(0,1)$, and $r \geq 0, \mathcal{C}^{r, k}(I)$ will denote the space of functions whose $r$ th derivatives are Hölder continuous with exponent $k$, equipped with the norm

$$
\|v\|_{r, k}=\max _{0 \leq k \leq r} \max _{x \in I}\left|\partial_{x}^{k} v(x)\right|+\sup _{\substack{x, y \in I \\ x \neq y}} \frac{\left|\partial_{x}^{r} v(x)-\partial_{x}^{k} v(y)\right|}{|x-y|^{k}} .
$$

LEMma 3.1 (see [15]). Let $I_{N}^{\alpha, \beta} v$ be the interpolation operator associated with the Gauss-Jacobi points, then for any function $v \in H_{w}^{m}(I)$, there exists a positive 
constant $C$ independent of $N$ such that

$$
\begin{aligned}
& \left\|v-I_{N}^{\alpha, \beta} v\right\|_{\infty} \leq C N^{\frac{1}{2}-m}|v|_{H_{w}^{m}(I)}, \\
& \left\|v-I_{N}^{\alpha, \beta} v\right\|_{w^{\alpha, \beta}} \leq C N^{-m}|v|_{H_{w}^{m}(I)} .
\end{aligned}
$$

Lemma 3.2 (see [14]). Let $\left\{L_{j}\right\}_{j=0}^{N}$ be the Nth Lagrange interpolation polynomials associated with the Gauss-Jacobi points. Then

$$
\left\|I_{N}^{\alpha, \beta}\right\|_{\infty}=\max _{x \in I} \sum_{j=0}^{N}\left|L_{j}(x)\right|= \begin{cases}\mathcal{O}(\log N) & \text { if }-1<\alpha, \beta \leq-\frac{1}{2}, \\ \mathcal{O}\left(N^{\frac{1}{2}+\max \{\alpha, \beta\}}\right) & \text { if otherwise. }\end{cases}
$$

LEMma 3.3 (see [15]). For every bounded function $v(x)$, there exists a positive constant $C$ independent of $v$ such that

$$
\left\|I_{N}^{\alpha, \beta} v\right\|_{w^{\alpha, \beta}} \leq C\|v\|_{\infty} .
$$

Lemma 3.4 (see [6]). Let $v \in H_{w}^{m}(I)$ for $m>\frac{1}{2}$, there exists a positive constant $C$ independent of $N$ such that

$$
\left|\langle v, \phi\rangle-\langle v, \phi\rangle_{N}\right| \leq C N^{-m}|v|_{H_{w}^{m}(I)}\|\phi\|_{w^{\alpha, \beta}}, \quad \forall \phi \in \mathcal{P}_{N},
$$

where

$$
\langle v, \phi\rangle=\int_{-1}^{1} v(x) \phi(x) d x \quad \text { and } \quad\langle v, \phi\rangle_{N}=\sum_{j=0}^{N} v\left(x_{j}\right) \phi\left(x_{j}\right) w_{j} .
$$

Lemma 3.5 (see [10]). Suppose that $H \geq 0$, let $E(x)$ be a non-negative integrable function satisfying

$$
E(x) \leq H \int_{-1}^{x} E(y) d y+G(x), \quad x \in I
$$

where $G(x)$ is also an integrable function, then there exists a positive constant $C$ such that

$$
\begin{aligned}
\|E\|_{w^{\alpha, \beta}} & \leq C\|G\|_{w^{\alpha, \beta}}, \\
\|E\|_{\infty} & \leq C\|G\|_{\infty} .
\end{aligned}
$$

Lemma 3.6 (see [18]). For a nonnegative integer $r$ and $k \in(0,1)$, there exists a constant $C_{r, k}>0$, such that for any function $v \in \mathcal{C}^{r, k}(I)$, there exists a polynomial function $T_{N} v \in \mathcal{P}_{N}$ such that

$$
\left\|v-T_{N} v\right\|_{\infty} \leq C_{r, k} N^{-(r+k)}\|v\|_{r, k} .
$$

Lemma 3.7 (see [7]). Let $\mathcal{A}_{v}$ be the operator defined by

$$
\mathcal{A}_{v}(x)=\int_{-1}^{x} \tilde{V}(x, t)(v(t)) d t
$$


then for any function $v \in \mathcal{C}(I)$, there exists a positive constant $C$ such that

$$
\left\|\mathcal{A}_{v}\right\|_{0, k} \leq C\|v\|_{\infty}, \quad 0<k<1 .
$$

Remark 1. From all the following, we assume that the nonlinear functions $\psi_{1}$ and $\psi_{2}$ and all its derivatives up to order $m$ satisfy a local Lipschitz condition such that for every $v_{1}, v_{2} \in \mathcal{C}(I)$

$$
\left|\frac{\partial^{k}}{\partial y^{k}} \psi_{i}\left(v_{1}\right)-\frac{\partial^{k}}{\partial y^{k}} \psi_{i}\left(v_{2}\right)\right| \leq L_{i k}\left|v_{1}-v_{2}\right|, \quad i=1,2 . \quad k=1,2, \ldots, m,
$$

we also consider that

$$
L=\max _{1 \leq k \leq m} L_{i k}
$$

\subsection{Error estimate in $L^{\infty}$-norm}

ThEOREM 3.8. Let $U$ be the exact solution of the nonlinear Volterra-Fredholm integral equation (2), which is assumed to be sufficiently smooth. Let the approximated solution $U_{N}$ be obtained by using the spectral scheme (7) and suppose that in Eq. (10) the nonlinear functions $\psi_{1}$ and $\psi_{2}$ and all its derivatives up to order $m$ satisfy a local Lipschitz condition. Then there is a positive constant $C$ such that the errors satisfy for $m \geq 1$,

where

$$
\left\|U-U_{N}\right\|_{\infty} \leq C N^{\frac{1}{2}-m}\left(|U|_{H_{w}^{m}(I)}+\chi\left\|I_{N}^{\alpha, \beta}\right\|_{\infty}\right)
$$

$$
\begin{gathered}
\chi=\|\widetilde{V}\|_{\infty}\left|\psi_{1}(U)\right|_{H_{w}^{m}(I)}+\|\widetilde{F}\|_{\infty}\left|\psi_{2}(U)\right|_{H_{w}^{m}(I)}+ \\
N^{-\frac{1}{2}}\left(\vartheta_{m, N}^{\alpha, \beta}\left\|\psi_{1}(U)\right\|_{\infty}+\tau_{m, N}^{\alpha, \beta}\left\|\psi_{2}(U)\right\|_{\infty}\right), \\
\vartheta_{m, N}^{\alpha, \beta}=\max _{0 \leq j \leq N}\left|\widetilde{V}\left(\sigma_{N, j}^{\alpha, \beta}, \cdot\right)\right|_{H_{w}^{m}(I)}, \quad \tau_{m, N}^{\alpha, \beta}=\max _{0 \leq j \leq N}\left|\widetilde{F}\left(\sigma_{N, j}^{\alpha, \beta}, \cdot\right)\right|_{H_{w}^{m}(I)} .
\end{gathered}
$$

P r o o f. At the collocation points $x=\sigma_{N, j}^{\alpha, \beta}$, we have $U_{N}\left(\sigma_{N, j}^{\alpha, \beta}\right)=\hat{U}_{N, j}^{\alpha, \beta}$.

We subtract (7) from (3) to obtain

$$
\begin{aligned}
U\left(\sigma_{N, j}^{\alpha, \beta}\right)-U_{N}\left(\sigma_{N, j}^{\alpha, \beta}\right)= & \left\langle\widetilde{V}\left(\sigma_{N, j}^{\alpha, \beta}, \theta\right), \psi_{1}\left(U\left(y_{N, j}^{\alpha, \beta}(\theta)\right)\right)\right\rangle \\
& +\left\langle\widetilde{F}\left(\sigma_{N, j}^{\alpha, \beta}, \theta\right), \psi_{2}(U(\theta))\right\rangle \\
& -\left\langle\widetilde{V}\left(\sigma_{N, j}^{\alpha, \beta}, \cdot\right), I_{N}^{\alpha, \beta} \psi_{1}\left(U_{N}\left(y_{N, j}^{\alpha, \beta}(\cdot)\right)\right)\right\rangle_{N} \\
& -\left\langle\widetilde{F}\left(\sigma_{N, j}^{\alpha, \beta}, \cdot\right), I_{N}^{\alpha, \beta} \psi_{2}\left(U_{N}(\cdot)\right)\right\rangle_{N} \\
= & \left\langle\widetilde{V}\left(\sigma_{N, j}^{\alpha, \beta}, \theta\right), \psi_{1}\left(U\left(y_{N, j}^{\alpha, \beta}(\theta)\right)\right)-\psi_{1}\left(U_{N}\left(y_{N, j}^{\alpha, \beta}(\theta)\right)\right)\right\rangle \\
& +\left\langle\widetilde{F}\left(\sigma_{N, j}^{\alpha, \beta}, \theta\right), \psi_{2}(U(\theta))-\psi_{2}\left(U_{N}(\theta)\right)\right\rangle+J\left(\sigma_{N, j}^{\alpha, \beta}\right),
\end{aligned}
$$


where

$$
\begin{aligned}
J\left(\sigma_{N, j}^{\alpha, \beta}\right)= & \left\langle\widetilde{V}\left(\sigma_{N, j}^{\alpha, \beta}, \theta\right), \psi_{1}\left(U_{N}\left(y_{N, j}^{\alpha, \beta}(\theta)\right)\right)\right\rangle \\
& -\left\langle\widetilde{V}\left(\sigma_{N, j}^{\alpha, \beta}, \cdot\right), I_{N}^{\alpha, \beta} \psi_{1}\left(U_{N}\left(y_{N, j}^{\alpha, \beta}(\cdot)\right)\right)\right\rangle_{N} \\
& +\left\langle\widetilde{F}\left(\sigma_{N, j}^{\alpha, \beta}, \theta\right), \psi_{2}\left(U_{N}(\theta)\right)\right\rangle-\left\langle\widetilde{F}\left(\sigma_{N, j}^{\alpha, \beta}, \cdot\right), I_{N}^{\alpha, \beta} \psi_{2}\left(U_{N}(\cdot)\right)\right\rangle_{N} \cdot
\end{aligned}
$$

Let the error function be written as

$$
E(x)=U(x)-U_{N}(x),
$$

then

$$
\begin{aligned}
E\left(\sigma_{N, j}^{\alpha, \beta}\right)= & \left\langle\widetilde{V}\left(\sigma_{N, j}^{\alpha, \beta}, \theta\right), \psi_{1}\left(U\left(y_{N, j}^{\alpha, \beta}(\theta)\right)\right)-\psi_{1}\left(U_{N}\left(y_{N, j}^{\alpha, \beta}(\theta)\right)\right)\right\rangle \\
& +\left\langle\widetilde{F}\left(\sigma_{N, j}^{\alpha, \beta}, \theta\right), \psi_{2}(U(\theta))-\psi_{2}\left(U_{N}(\theta)\right)\right\rangle+J\left(\sigma_{N, j}^{\alpha, \beta}\right)
\end{aligned}
$$

multiplying $L_{j}$ on both sides of (21) and summing up from $j=0$ to $N$, yield

$$
I_{N}^{\alpha, \beta}\left(U(x)-U_{N}(x)\right)=I_{N}^{\alpha, \beta} Q_{1}(x)+I_{N}^{\alpha, \beta} Q_{2}(x)+\sum_{j=0}^{N} J\left(\sigma_{N, j}^{\alpha, \beta}\right) L_{j}(x),
$$

where

$$
Q_{1}(x)=\int_{-1}^{x} \tilde{V}(x, y)\left[\psi_{1}(U(y))-\psi_{1}\left(U_{N}(y)\right)\right] d y
$$

and

$$
Q_{2}(x)=\int_{-1}^{1} \widetilde{F}(x, y)\left[\psi_{2}(U(y))-\psi_{2}\left(U_{N}(y)\right)\right] d y
$$

By adding and subtracting $Q_{1}(x)$ and $U(x)$ into the right-hand side of (22) we get

$$
\begin{aligned}
|E(x)| \leq & \int_{-1}^{x}\left|\widetilde{V}(x, y)\left[\psi_{1}(U(y))-\psi_{1}\left(U_{N}(y)\right)\right]\right| d y+\left|U(x)-I_{N}^{\alpha, \beta}(U(x))\right| \\
& +\left|Q_{1}(x)-I_{N}^{\alpha, \beta} Q_{1}(x)\right|+\left|I_{N}^{\alpha, \beta} Q_{2}(x)\right|+\left|\sum_{j=0}^{N} J\left(\sigma_{N, j}^{\alpha, \beta}\right) L_{j}(x)\right| \\
\leq & \max _{(x, y) \in I \times I}|\widetilde{V}(x, y)| \int_{-1}^{x}|E(y)| d y+\sum_{j=1}^{4} I_{j}(x),
\end{aligned}
$$


where

$$
\begin{aligned}
& I_{1}(x)=\left|U(x)-I_{N}^{\alpha, \beta}(U(x))\right|, \\
& I_{2}(x)=\sum_{j=0}^{N}\left|J\left(\sigma_{N, j}^{\alpha, \beta}\right) L_{j}(x)\right|, \\
& I_{3}(x)=\left|Q_{1}(x)-I_{N}^{\alpha, \beta} Q_{1}(x)\right|, \\
& I_{4}(x)=\left|I_{N}^{\alpha, \beta} Q_{2}(x)\right| .
\end{aligned}
$$

Then the Gronwall inequality (16) gives

$$
\|E\|_{\infty} \leq C\left(\left\|I_{1}\right\|_{\infty}+\left\|I_{2}\right\|_{\infty}+\left\|I_{3}\right\|_{\infty}+\left\|I_{4}\right\|_{\infty}\right) .
$$

Due to (10) we have

$$
\left\|I_{1}\right\|_{\infty}=\left\|U-I_{N}^{\alpha, \beta} U\right\|_{\infty} \leq C N^{\frac{1}{2}-m}|U|_{H_{w}^{m}(I)} .
$$

From (20) we have

$$
\begin{aligned}
\left|J\left(\sigma_{N, j}^{\alpha, \beta}\right)\right| \leq & \\
\mid\left\langle\widetilde{V}\left(\sigma_{N, j}^{\alpha, \beta}, \theta\right), \psi_{1}(\right. & \left.\left.U_{N}\left(y_{N, j}^{\alpha, \beta}(\theta)\right)\right)\right\rangle-\left\langle\widetilde{V}\left(\sigma_{N, j}^{\alpha, \beta}, \cdot\right), I_{N}^{\alpha, \beta} \psi_{1}\left(U_{N}\left(y_{N, j}^{\alpha, \beta}(\cdot)\right)\right)\right\rangle_{N} \mid+ \\
& \left|\left\langle\widetilde{F}\left(\sigma_{N, j}^{\alpha, \beta}, \theta\right), \psi_{2}\left(U_{N}(\theta)\right)\right\rangle-\left\langle\widetilde{F}\left(\sigma_{N, j}^{\alpha, \beta}, \cdot\right), I_{N}^{\alpha, \beta} \psi_{2}\left(U_{N}(\cdot)\right)\right\rangle_{N}\right| .
\end{aligned}
$$

Thus, the right-hand side of the above inequality is less or equal to

$$
\begin{aligned}
& \left|\left\langle\widetilde{V}\left(\sigma_{N, j}^{\alpha, \beta}, \theta\right), \psi_{1}\left(U_{N}\left(y_{N, j}^{\alpha, \beta}(\theta)\right)\right)-I_{N}^{\alpha, \beta} \psi_{1}\left(U_{N}\left(y_{N, j}^{\alpha, \beta}(\cdot)\right)\right)\right\rangle\right| \\
& +\left|\left\langle\widetilde{V}\left(\sigma_{N, j}^{\alpha, \beta}, \theta\right), I_{N}^{\alpha, \beta} \psi_{1}\left(U_{N}\left(y_{N, j}^{\alpha, \beta}(\theta)\right)\right)\right\rangle-\left\langle\widetilde{V}\left(\sigma_{N, j}^{\alpha, \beta}, \cdot\right), I_{N}^{\alpha, \beta} \psi_{1}\left(U_{N}\left(y_{N, j}^{\alpha, \beta}(\cdot)\right)\right)\right\rangle_{N}\right| \\
& +\mid\left\langle\widetilde{F}\left(\sigma_{N, j}^{\alpha, \beta}, \theta\right), \psi_{2}\left(U_{N}(\theta)\right)-I_{N}^{\alpha, \beta} \psi_{2}\left(U_{N}(\theta)\right\rangle\right| \\
& +\left|\left\langle\widetilde{F}\left(\sigma_{N, j}^{\alpha, \beta}, \theta\right), I_{N}^{\alpha, \beta} \psi_{2}\left(U_{N}(\theta)\right)\right\rangle-\left\langle\widetilde{F}\left(\sigma_{N, j}^{\alpha, \beta}, \cdot\right), I_{N}^{\alpha, \beta} \psi_{2}\left(U_{N}(\cdot)\right)\right\rangle_{N}\right|,
\end{aligned}
$$

hence by using (14) we have

$$
\begin{gathered}
\max _{0 \leq j \leq N}\left|J\left(\sigma_{N, j}^{\alpha, \beta}\right)\right| \leq 2\|\widetilde{V}\|_{\infty}\left\|\psi_{1}\left(U_{N}\right)-I_{N}^{\alpha, \beta} \psi_{1}\left(U_{N}\right)\right\|_{\infty}+ \\
C N^{-m} \max _{0 \leq j \leq N}\left|\widetilde{V}\left(\sigma_{N, j}^{\alpha, \beta}, \cdot\right)\right|_{H_{w}^{m}(I)} \max _{0 \leq j \leq N}\left\|I_{N}^{\alpha, \beta} \psi_{1}\left(U_{N}\left(y_{N, j}^{\alpha, \beta}(\theta)\right)\right)\right\|_{w^{\alpha, \beta}}+ \\
2\|\widetilde{F}\|_{\infty}\left\|\psi_{2}\left(U_{N}\right)-I_{N}^{\alpha, \beta} \psi_{2}\left(U_{N}\right)\right\|_{\infty}+ \\
C N^{-m} \max _{0 \leq j \leq N}\left|\widetilde{F}\left(\sigma_{N, j}^{\alpha, \beta}, \cdot\right)\right|_{H_{w}^{m}(I)}\left\|I_{N}^{\alpha, \beta} \psi_{2}\left(U_{N}\right)\right\|_{w^{\alpha, \beta}} .
\end{gathered}
$$


From (10) we obtain

$$
\begin{gathered}
\max _{0 \leq j \leq N}\left|J\left(\sigma_{N, j}^{\alpha, \beta}\right)\right| \leq C N^{\frac{1}{2}-m}\|\widetilde{V}\|_{\infty}\left|\psi_{1}\left(U_{N}\right)\right|_{H_{w}^{m}(I)}+ \\
C N^{-m} \max _{0 \leq j \leq N}\left|\widetilde{V}\left(\sigma_{N, j}^{\alpha, \beta}, \cdot\right)\right|_{H_{w}^{m}(I)} \max _{0 \leq j \leq N}\left\|\psi_{1}\left(U_{N}\left(y_{N, j}^{\alpha, \beta}(\theta)\right)\right)\right\|_{\infty}+ \\
C N^{\frac{1}{2}-m}\|\widetilde{F}\|_{\infty}\left|\psi_{2}\left(U_{N}\right)\right|_{H_{w}^{m}(I)}+ \\
C N^{-m} \max _{0 \leq j \leq N}\left|\widetilde{F}\left(\sigma_{N, j}^{\alpha, \beta}, \cdot\right)\right|_{H_{w}^{m}(I)}\left\|\psi_{2}\left(U_{N}\right)\right\|_{\infty} .
\end{gathered}
$$

From the definition of semi-norm (8) we have

$$
\begin{aligned}
\left|\psi_{1}\left(U_{N}\right)\right|_{H_{w}^{m}(I)} & =\left|\psi_{1}\left(U_{N}\right)-\psi_{1}(U)+\psi_{1}(U)\right|_{H_{w}^{m}(I)} \\
& \leq\left|\psi_{1}(U)-\psi_{1}\left(U_{N}\right)\right|_{H_{w}^{m}(I)}+\left|\psi_{1}(U)\right|_{H_{w}^{m}(I)} \\
& \leq\left(\sum_{k=\min (m, N+1)}^{m} \|\left.\frac{\partial^{k} \psi_{1}}{\partial y^{k}}\left(U-U_{N}\right)\right|_{w^{\alpha, \beta}} ^{2}\right)^{1 / 2} \\
& +\left|\psi_{1}(U)\right|_{H_{w}^{m}(I)} .
\end{aligned}
$$

Since the nonlinear function $\psi_{1}$ and its derivatives of orders $1, \ldots, m$ satisfy the Lipschitz condition, we have

$$
\begin{aligned}
& \left\|\frac{\partial^{k} \psi_{1}}{\partial y^{k}}\left(U-U_{N}\right)\right\|_{w^{\alpha, \beta}}^{2} \\
& \leq L\left\|\left(U-U_{N}\right)\right\|_{w^{\alpha, \beta}}^{2}, \quad \min (m, N+1) \leq k \leq m,
\end{aligned}
$$

hence

$$
\begin{aligned}
\left|\psi_{1}\left(U_{N}\right)\right|_{H_{w}^{m}(I)} & \leq L^{\prime}\|E\|_{w^{\alpha, \beta}}+\left|\psi_{1}(U)\right|_{H_{w}^{m}(I)} \\
& \leq L^{\prime \prime}\|E\|_{\infty}+\left|\psi_{1}(U)\right|_{H_{w}^{m}(I)}
\end{aligned}
$$

where $L^{\prime}$ and $L^{\prime \prime}$ are positive constants. Similarly, we can obtain

$$
\begin{aligned}
\left|\psi_{2}\left(U_{N}\right)\right|_{H_{w}^{m}(I)} & \leq L^{\prime}\|E\|_{w^{\alpha, \beta}}+\left|\psi_{2}(U)\right|_{H_{w}^{m}(I)} \\
& \leq L^{\prime \prime}\|E\|_{\infty}+\left|\psi_{2}(U)\right|_{H_{w}^{m}(I)}
\end{aligned}
$$


Therefore, combining (30), (31), (32), with (12) yields

$$
\begin{aligned}
\left\|I_{2}\right\|_{\infty}= & \left\|\sum_{j=0}^{N}\left|J\left(\sigma_{N, j}^{\alpha, \beta}\right)\right| L_{j}(x)\right\|_{\infty} \leq \max _{0 \leq j \leq N}\left|J\left(\sigma_{N, j}^{\alpha, \beta}\right)\right|\left\|I_{N}^{\alpha, \beta}\right\|_{\infty} \\
\leq & C N^{\frac{1}{2}-m}\|\widetilde{V}\|_{\infty}\left(L^{\prime \prime}\|E\|_{\infty}+\left|\psi_{1}(U)\right|_{H_{w}^{m}(I)}\right)\left\|I_{N}^{\alpha, \beta}\right\|_{\infty} \\
& +C N^{-m} \max _{0 \leq j \leq N}\left|\widetilde{V}\left(\sigma_{N, j}^{\alpha, \beta}, \cdot\right)\right|_{H_{w}^{m}(I)}\left(L\|E\|_{\infty}+\left\|\psi_{1}(U)\right\|_{\infty}\right)\left\|I_{N}^{\alpha, \beta}\right\|_{\infty} \\
& +C N^{\frac{1}{2}-m}\|\widetilde{F}\|_{\infty}\left(L^{\prime \prime}\|E\|_{\infty}+\left|\psi_{2}(U)\right|_{H_{w}^{m}(I)}\right)\left\|I_{N}^{\alpha, \beta}\right\|_{\infty} \\
& +C N^{-m} \max _{0 \leq j \leq N}\left|\widetilde{F}\left(\sigma_{N, j}^{\alpha, \beta}, \cdot\right)\right|_{H_{w}^{m}(I)}\left(L\|E\|_{\infty}+\left\|\psi_{2}(U)\right\|_{\infty}\right)\left\|I_{N}^{\alpha, \beta}\right\|_{\infty} .
\end{aligned}
$$

Let $\mathcal{A}_{v}$ be the operator defined in Lemma 3.7 and we consider

$$
v(x)=\psi_{1}(U(x))-\psi_{1}\left(U_{N}(x)\right) .
$$

From (17) and (18) we obtain

$$
\begin{aligned}
\left\|I_{3}\right\|_{\infty} & =\left\|\mathcal{A}_{v}\left(I-I_{N}^{\alpha, \beta}\right)\right\|_{\infty} \\
& =\left\|\left(\mathcal{A}_{v}-T_{N} \mathcal{A}_{v}\right)\left(I-I_{N}^{\alpha, \beta}\right)\right\|_{\infty} \\
& \leq\left\|\mathcal{A}_{v}-T_{N} \mathcal{A}_{v}\right\|_{\infty}\left\|I-I_{N}^{\alpha, \beta}\right\|_{\infty} \\
& \leq C_{0, k} N^{-k}\left\|\mathcal{A}_{v}\right\|_{0, k}\left(1+\left\|I_{N}^{\alpha, \beta}\right\|_{\infty}\right) \\
& \leq C_{0, k} N^{-k}\|v\|_{\infty}\left(1+\left\|I_{N}^{\alpha, \beta}\right\|_{\infty}\right) \\
& \leq C_{0, k} N^{-k}\left\|\psi_{1}(U(x))-\psi_{1}\left(U_{N}(x)\right)\right\|_{\infty}\left(1+\left\|I_{N}^{\alpha, \beta}\right\|_{\infty}\right) \\
& \leq C_{0, k} L N^{-k}\|E\|_{\infty}\left(1+\left\|I_{N}^{\alpha, \beta}\right\|_{\infty}\right) .
\end{aligned}
$$

Finally, we have

$$
\begin{aligned}
\left\|I_{4}\right\|_{\infty} & =\left\|I_{N}^{\alpha, \beta} Q_{2}\right\|_{\infty} \leq \max _{0 \leq j \leq N}\left|Q_{2}\left(\sigma_{N, j}^{\alpha, \beta}\right)\right|\left\|I_{N}^{\alpha, \beta}\right\|_{\infty} \\
& \leq \max _{0 \leq j \leq N}\left|\int_{-1}^{1} \widetilde{F}\left(\sigma_{N, j}^{\alpha, \beta}, y\right)\left[\psi_{2}(U(y))-\psi_{2}\left(U_{N}(y)\right)\right] d y\right|\left\|I_{N}^{\alpha, \beta}\right\|_{\infty} \\
& \leq 2 L\|\widetilde{F}\|_{\infty}\|E\|_{\infty}\left\|I_{N}^{\alpha, \beta}\right\|_{\infty} .
\end{aligned}
$$

Combining (29), (33), (34), (35) with (28) gives the desired estimate (19). 


\section{F. HAMANI - A. RAHMOUNE}

\subsection{Error estimate in $L_{w^{\alpha, \beta}}^{2}-$ norm}

Theorem 3.9. Assume that the hypotheses in Theorem 3.8 hold, and $k \in(0,1)$. Then

$$
\begin{aligned}
& \left\|U-U_{N}\right\|_{w^{\alpha, \beta}} \\
& \leq C N^{-m}\left(|U|_{H_{w}^{m}(I)}+N^{-\frac{1}{2}} \chi+\rho\left(|U|_{H_{w}^{m}(I)}+\chi\left\|I_{N}^{\alpha, \beta}\right\|_{\infty}\right)\right),
\end{aligned}
$$

where $\vartheta_{m, N}^{\alpha, \beta}, \tau_{m, N}^{\alpha, \beta}$ and $\chi$ are given as in Theorem 3.8 and

$$
\rho=N^{\frac{1}{2}}\|\widetilde{F}\|_{\infty}+N^{\frac{1}{2}-k}+N^{\frac{1}{2}-m}\left(\vartheta_{m, N}^{\alpha, \beta}+\tau_{m, N}^{\alpha, \beta}\right) .
$$

P r o of. By using (23) and the Gronwall inequality (15) we have

$$
\|E\|_{w^{\alpha, \beta}} \leq C\left(\left\|I_{1}\right\|_{w^{\alpha, \beta}}+\left\|I_{2}\right\|_{w^{\alpha, \beta}}+\left\|I_{3}\right\|_{w^{\alpha, \beta}}+\left\|I_{4}\right\|_{w^{\alpha, \beta}}\right) .
$$

From (13) we obtain

By Lemma 3.3 we have

$$
\begin{aligned}
\left\|I_{1}\right\|_{w^{\alpha, \beta}} & =\left\|U-I_{N}^{\alpha, \beta} U\right\|_{w^{\alpha, \beta}} \\
& \leq C N^{-m}|U|_{H_{w}^{m}(I)} .
\end{aligned}
$$

$$
\begin{aligned}
\left\|I_{2}\right\|_{w^{\alpha, \beta}} & =\left\|\sum_{j=0}^{N} J\left(\sigma_{N, j}^{\alpha, \beta}\right) L_{j}(x)\right\|_{w^{\alpha, \beta}} \\
& \leq C\|J\|_{\infty} .
\end{aligned}
$$

Combining (30), (31), (32) leads

$$
\begin{aligned}
\left\|I_{2}\right\|_{w^{\alpha, \beta}} \leq & C N^{\frac{1}{2}-m}\|\widetilde{V}\|_{\infty}\left(L^{\prime \prime}\|E\|_{w^{\alpha, \beta}}+\left|\psi_{1}(U)\right|_{H_{w}^{m}(I)}\right) \\
& +C N^{-m} \max _{0 \leq j \leq N}\left|\widetilde{V}\left(\sigma_{N, j}^{\alpha, \beta}, \cdot\right)\right|_{H_{w}^{m}(I)}\left(L\|E\|_{\infty}+\left\|\psi_{1}(U)\right\|_{\infty}\right) \\
& +C N^{\frac{1}{2}-m}\|\widetilde{F}\|_{\infty}\left(L^{\prime \prime}\|E\|_{w^{\alpha, \beta}}+\left|\psi_{2}(U)\right|_{H_{w}^{m}(I)}\right) \\
& +C N^{-m} \max _{0 \leq j \leq N}\left|\widetilde{F}\left(\sigma_{N, j}^{\alpha, \beta}, \cdot\right)\right|_{H_{w}^{m}(I)}\left(L\|E\|_{\infty}+\left\|\psi_{2}(U)\right\|_{\infty}\right) .
\end{aligned}
$$

Let us consider

$$
v(x)=\psi_{1}(U(x))-\psi_{1}\left(U_{N}(x)\right),
$$


similarly to the estimate of $\left\|I_{3}\right\|_{\infty}$ and by using (17) and (18) we have

$$
\begin{aligned}
\left\|I_{3}\right\|_{w^{\alpha, \beta}} & =\left\|\left(I_{N}^{\alpha, \beta}-I\right) \mathcal{A}_{v}\right\|_{w^{\alpha, \beta}} \\
& =\left\|\left(I-I_{N}^{\alpha, \beta}\right)\left(\mathcal{A}_{v}-T_{N} \mathcal{A}_{v}\right)\right\|_{w^{\alpha, \beta}} \\
& \leq\left\|\mathcal{A}_{v}-T_{N} \mathcal{A}_{v}\right\|_{w^{\alpha, \beta}}+\left\|I_{N}^{\alpha, \beta}\left(\mathcal{A}_{v}-T_{N} \mathcal{A}_{v}\right)\right\|_{w^{\alpha, \beta}} \\
& \leq C\left\|\mathcal{A}_{v}-T_{N} \mathcal{A}_{v}\right\|_{\infty} \\
& \leq C N^{-k}\left\|\mathcal{A}_{v}\right\|_{0, k} \\
& \leq C N^{-k}\|v\|_{\infty} \\
& \leq C N^{-k}\left\|\psi_{1}(U)-\psi_{1}\left(U_{N}\right)\right\|_{\infty} \\
& \leq C L N^{-k}\|E\|_{\infty} .
\end{aligned}
$$

From (13) we obtain

$$
\begin{aligned}
\left\|I_{4}\right\|_{w^{\alpha, \beta}} & =\left\|I_{N}^{\alpha, \beta} Q_{2}\right\|_{w^{\alpha, \beta}} \\
& \leq C\left\|Q_{2}\right\|_{\infty} \\
& \leq C \max _{0 \leq j \leq N}\left|Q_{2}\left(\sigma_{N, j}^{\alpha, \beta}\right)\right| \\
& \leq C \max _{0 \leq j \leq N}\left|\int_{-1}^{1} \widetilde{F}\left(\sigma_{N, j}^{\alpha, \beta}, y\right)\left[\psi_{2}(U(y))-\psi_{2}\left(U_{N}(y)\right)\right] d y\right| \\
& \leq C L\|\widetilde{F}\|_{\infty}\|E\|_{\infty} .
\end{aligned}
$$

The desired estimate (36) is obtained by combining (38)-(42), (37) and taking into account the convergence result in Theorem 3.8 .

\section{Numerical examples}

In this section, some illustrative examples are provided to demonstrate the applicability of the designed method. The calculations performed in the examples are calculated by the Matlab software, and a Core i5-2520M CPU 2.5 GHZ and 4 GB RAM are used to run the programs. 
ExAMPLE 1. Consider the nonlinear Volterra-Fredholm integral equation,

with

$$
u(s)=g(s)+\int_{0}^{s}(s-t) u^{2}(t) d t+\int_{0}^{1}(s+t) u(t) d t, \quad s \in[0,1],
$$

$$
g(s)=-\frac{1}{30} s^{6}+\frac{1}{3} s^{4}-s^{2}+\frac{5}{3} s-\frac{5}{4},
$$

the exact solution of which is

$$
u(s)=s^{2}-2 .
$$

Table 1 shows the numerical errors obtained by using the spectral method described above with $\alpha=\beta=-3 / 4$. Table 2 shows the comparison of the absolute errors of this method for $N=5$ at some equally-spaced points on $[0,1]$ with those previously obtained by using three other collocation methods: The first one is based on Chebyshev approximation [3], the second one on rationalized Haar functions ( $\mathrm{RH})$ [16, while the third one on multiquadrics radial basis functions (MQ-RBFs) [17] in which the zeros of the shifted Legendre polynomial are chosen as collocation points. We observe that the numerical results are in good accordance with the theoretical analysis and we can see that the proposed method is better than the considered literature methods.

TABLE 1. The $L^{\infty}$ and $L_{w}^{2}$ errors for Example 1

\begin{tabular}{|c||c|c|c|c|}
\hline$N$ & 2 & 4 & 6 & 8 \\
\hline$L^{\infty}$ Error & $6.0526 \mathrm{e}-02$ & $4.5375 \mathrm{e}-04$ & $1.3323 \mathrm{e}-15$ & $1.5543 \mathrm{e}-15$ \\
$L_{w}^{2}$ Error & $9.7168 \mathrm{e}-02$ & $2.7076 \mathrm{e}-04$ & $9.3726 \mathrm{e}-16$ & $8.2961 \mathrm{e}-16$ \\
\hline
\end{tabular}

Example 2. Consider the Volterra integral equation

$$
u(s)=s+\cos s-1+\int_{0}^{s} \sin (u(t)) d t, \quad s \in[0,1],
$$

where $u(s)=s$ is the exact solution. Table 3 shows the numerical errors for $\alpha=1 / 4$ and $\beta=1 / 3$. Table 4 shows a comparison of the absolute errors at some points with those obtained using fixed point technique and cubic B-spline wavelets [12]. 
TABLe 2. Absolute errors for Example 1

\begin{tabular}{|l||c|c|c|c|}
\hline \multicolumn{1}{|c||}{$s$} & $\begin{array}{c}\text { Present method } \\
\text { for } N=5\end{array}$ & $\begin{array}{c}\text { Method in [3] } \\
\text { for } N=5\end{array}$ & $\begin{array}{c}\text { Method in [16] } \\
\text { for } N=16\end{array}$ & $\begin{array}{c}\text { Method in [17] } \\
\text { for } N=10\end{array}$ \\
\hline 0 & $8.88 \mathrm{e}-16$ & $0.20 \mathrm{e}-9$ & $8.00 \mathrm{e}-6$ & $1.92 \mathrm{e}-8$ \\
0.2 & $4.44 \mathrm{e}-16$ & $7.35 \mathrm{e}-9$ & $4.00 \mathrm{e}-6$ & $4.16 \mathrm{e}-9$ \\
0.4 & $2.22 \mathrm{e}-16$ & $7.93 \mathrm{e}-9$ & $1.10 \mathrm{e}-5$ & $2.53 \mathrm{e}-9$ \\
0.6 & $0.00 \mathrm{e}+00$ & $2.55 \mathrm{e}-9$ & $1.30 \mathrm{e}-5$ & $1.92 \mathrm{e}-9$ \\
0.8 & $0.00 \mathrm{e}+00$ & $3.98 \mathrm{e}-9$ & $1.40 \mathrm{e}-5$ & $1.83 \mathrm{e}-9$ \\
1 & $6.66 \mathrm{e}-16$ & $2.64 \mathrm{e}-9$ & $1.40 \mathrm{e}-5$ & $5.07 \mathrm{e}-9$ \\
\hline
\end{tabular}

TABLE 3. The $L^{\infty}$ and $L_{w}^{2}$ errors for Example 2

\begin{tabular}{|c||c|c|c|c|c|}
\hline$N$ & 2 & 4 & 6 & 8 & 10 \\
\hline$L^{\infty}$ error & $5.1387 \mathrm{e}-03$ & $1.8428 \mathrm{e}-05$ & $2.7832 \mathrm{e}-08$ & $2.3683 \mathrm{e}-11$ & $1.2546 \mathrm{e}-14$ \\
$L_{w}^{2}$ error & $2.7245 \mathrm{e}-03$ & $9.1760 \mathrm{e}-06$ & $1.3662 \mathrm{e}-08$ & $1.1807 \mathrm{e}-11$ & $6.5577 \mathrm{e}-15$ \\
\hline
\end{tabular}

TABLE 4. Absolute errors for Example 2

\begin{tabular}{|l||c|c|}
\hline \multicolumn{1}{|c||}{$s$} & $\begin{array}{c}\text { Present method } \\
\text { for } N=10\end{array}$ & $\begin{array}{c}\text { Method of [12] } \\
\text { for } N=10\end{array}$ \\
\hline 0.2 & $5.38 \mathrm{e}-14$ & $0.00 \mathrm{e}+00$ \\
0.4 & $4.69 \mathrm{e}-15$ & $4.22 \mathrm{e}-08$ \\
0.6 & $1.08 \mathrm{e}-14$ & $1.09 \mathrm{e}-08$ \\
0.8 & $5.55 \mathrm{e}-16$ & $2.35 \mathrm{e}-08$ \\
1 & $6.66 \mathrm{e}-15$ & $1.42 \mathrm{e}-08$ \\
$3.56 \mathrm{e}-14$ & $2.63 \mathrm{e}-08$ \\
\hline
\end{tabular}


ExAmPLE 3. Consider the Fredholm integral equation

$$
u(s)=e^{s+1}-\int_{0}^{1} e^{s-2 t} u^{3}(t) d t, \quad s \in[0,1],
$$

where $u(s)=e^{s}$ is the exact solution. Table 5 shows the numerical errors for $\alpha=\beta=1 / 2$. Table 6 shows the absolute errors at some points compared with those obtained using Haar wavelets [4].

TABLE 5. The $L^{\infty}$ and $L_{w}^{2}$ errors for Example 3

\begin{tabular}{|c||c|c|c|c|c|}
\hline$N$ & 2 & 4 & 6 & 8 & 10 \\
\hline \hline$L^{\infty}$ error & $6.3352 \mathrm{e}-02$ & $2.4390 \mathrm{e}-03$ & $3.5130 \mathrm{e}-05$ & $2.8389 \mathrm{e}-07$ & $1.4804 \mathrm{e}-09$ \\
$L_{w}^{2}$ error & $5.0454 \mathrm{e}-02$ & $1.2316 \mathrm{e}-03$ & $1.7110 \mathrm{e}-05$ & $1.3598 \mathrm{e}-07$ & $7.0234 \mathrm{e}-10$ \\
\hline
\end{tabular}

TABLE 6. Absolute errors for Example 3

\begin{tabular}{|c||c|c|}
\hline$s$ & $\begin{array}{c}\text { Present method } \\
\text { for } N=10\end{array}$ & $\begin{array}{c}\text { Method of [4] } \\
\text { for } N=32\end{array}$ \\
\hline \hline 0.1 & $6.14 \mathrm{e}-10$ & $2.05 \mathrm{e}-03$ \\
0.3 & $7.50 \mathrm{e}-10$ & $8.69 \mathrm{e}-03$ \\
0.5 & $9.17 \mathrm{e}-10$ & $1.87 \mathrm{e}-02$ \\
0.7 & $1.12 \mathrm{e}-09$ & $2.93 \mathrm{e}-03$ \\
0.9 & $1.37 \mathrm{e}-09$ & $2.16 \mathrm{e}-02$ \\
\hline
\end{tabular}

\section{Conclusion}

In this paper, we have proposed an accurate spectral collocation method based on the Jacobi orthogonal polynomials for the solutions of nonlinear VolterraFredholm integral equations. The theorems on the convergence and error estimates of the method have been stated and proved in the $L^{\infty}$-norm and the weighted $L^{2}$-norm. Some numerical examples from the literature are provided to illustrate the efficiency and effectiveness of the proposed approach. 


\section{AN ACCURATE SPECTRAL METHOD FOR NONLINEAR INTEGRAL EQUATIONS}

\section{REFERENCES}

[1] AMIRI, S.-HAJIPOUR, M.-BALEANU, D.: A spectral collocation method with piecewise trigonometric basis functions for nonlinear Volterra-Fredholm integral equations, Appl. Math. Comput. 370 (2020), 124915.

[2] ASKEY, R.: Orthogonal Polynomials and Special Functions. Society for Industrial and Applied Mathematics, Philadelphia, PA, 1975.

[3] BABOLIAN, E.-FATTAHZADEH, F.-GOLPAR RABOKY, E.: A Chebyshev approximation for solving nonlinear integral equations of Hammerstein type, Appl. Math. Comput. 189 (2007), 641-646.

[4] BABOLIAN, E.-SHAHSAVARAN, A.: Numerical solution of nonlinear Fredholm integral equations of the second kind using Haar wavelets, J. Comput. Appl. Math. 225 (2009), 87-95.

[5] BENYOUSSEF, S.-RAHMOUNE, A.: Efficient spectral-collocation methods for a class of linear Fredholm integro-differential equations on the half-line, J. Comput. Appl. Math. 377 (2020), 112894.

[6] CAnuto, C.-Hussaini, M.-QUARTEROni, A.-ZANG, T.: Orthogonal Polynomials and Special Functions. (1 edition). Springer-Verlag Berlin Heidelberg, 2006.

[7] CHEN, Y.-TANG, T.: Convergence analysis of the Jacobi spectral collocation methods for Volterra integral equations with a weakly singular kernel, Math. Comp. 79 (2010), $147-167$.

[8] GUECHI, A.-RAHMOUNE, A.: Mapped Chebyshev spectral methods for solving second kind integral equations on the real line, Aust. J. Math. Anal. Appl. 14 (2017), 1-8.

[9] GUO, B.-Y.-SHEN, J.-WANG, L.-L.: Generalized Jacobi polynomials/functions and their applications, Appl. Numer. Math. 59 (2009), 1011-1028.

[10] HEADLEY, V. B.: A multidimensional nonlinear gronwall inequality, J. Math. Anal. Appl. 47 (1974), 250-255.

[11] MALEKNEJAD, K.-HASHEMIZADEH, E.-BASIRAT, B.: Computational method based on Bernstein operational matrices for nonlinear Volterra-Fredholm-Hammerstein integral equations, Commun. Nonlinear Sci. Numer. Simul. 17 (2012), 52-61.

[12] MALEKNEJAD, K.-MOLLAPOURASL, R.-SHAHABI, M.: On the solution of a nonlinear integral equation on the basis of a fixed point technique and cubic B-spline scaling functions, J. Comput. Appl. Math. 239 (2013), 346-358.

[13] MARZBAN, H.-TABRIZIDOOZ, H.-RAZZAGHI, M.: A composite collocation method for the nonlinear mixed Volterra-Fredholm-Hammerstein integral equations, Commun. Nonlinear Sci. Numer. Simul. 16 (2011), 1186-1194.

[14] MASTROIANNI, G.-OCCORSIO, D.: Optimal systems of nodes for Lagrange interpolation on bounded intervals. A survey, J. Comput. Appl. Math. 134 (2001), 325-341.

[15] NEVAI, P.: Mean convergence of lagrange interpolation, III, Trans. Amer. Math. Soc. 282 (1984), 669-698.

[16] ORDOKHANI, Y.-RAZZAGHI, M.: Solution of nonlinear Volterra-Fredholm-Hammerstein integral equations via a collocation method and rationalized Haar functions, Appl. Math. Lett. 21 (2008), 4-9. 


\title{
F. HAMANI - A. RAHMOUNE
}

[17] PARAND, K.-RAD, J. A.: Numerical solution of nonlinear Volterra-Fredholm-Hammerstein integral equations via collocation method based on radial basis functions, Appl. Math. Comput. 218 (2012), 5292-5309.

[18] RAGOZIN, D. L.: Polynomial approximation on compact manifolds and homogeneous spaces, Trans. Amer. Math. Soc. 150 (1970), 41-53.

[19] RAHMOUNE, A.: On the numerical solution of integral equations of the second kind over infinite intervals, J. Appl. Math. Comput. 66 (2021), 129-148.

[20] RAHMOUNE, A.-GUECHI, A.: A rational spectral collocation method for solving Fredholm integral equations on the whole line, Int. J. Comput. Sci. Math. 13 (2021), 32-41.

[21] YOUSEFI, S.-RAZZAGHI, M.: Legendre wavelets method for the nonlinear Volterra-Fredholm integral equations, Math. Comput. Simulation 70 (2005), 1-8.

Received February 16, 2021

\author{
Fatima, Hamani \\ Department of mathematics \\ University of M'sila \\ 28000 M'sila \\ ALGERIA \\ E-mail: fatima.hamani@univ-bba.dz \\ Azedine, Rahmoune \\ Department of mathematics \\ University of of Bordj Bou Arreridj \\ 34030 El Anasser \\ ALGERIA \\ E-mail: a.rahmoune@univ-bba.dz
}

